\title{
Extreme Learning Machine Based Prognostics of Battery Life
}

\author{
Roozbeh Razavi-Far*, Shiladitya Chakrabarti ${ }^{\dagger}$ and Mehrdad Saif ${ }^{\ddagger}$ \\ Department of Electrical and Computer Engineering, University of Windsor \\ Windsor, Ontario N9B $3 \mathrm{P}_{4}$ Canada \\ *roozbeh@uwindsor.ca \\ ${ }^{\dagger}$ chakrab@uwindsor.ca \\ ${ }^{\ddagger}$ msaif@uwindsor.ca \\ Enrico Zio \\ Ecole CentraleSupélec, Université Paris Saclay, France \\ Department of Energy, Politecnico di Milano, Milano, Italy \\ enrico.zio@ecp.fr; enrico.zio@supelec.fr; enrico.zio@polimi.it \\ Vasile Palade \\ School of Computing, Electronics and Mathematics \\ Coventry University, CV1 5FB Coventry, United Kingdom \\ ab5839@coventry.ac.uk
}

Received 13 February 2018

Accepted 5 November 2018

Published 20 December 2018

This paper presents a prognostic scheme for estimating the remaining useful life of Lithium-ion batteries. The proposed scheme utilizes a prediction module that aims to obtain precise predictions for both short and long prediction horizons. The prediction module makes use of extreme learning machines for one-step and multi-step ahead predictions, using various prediction strategies, including iterative, direct and DirRec, which use the constant-current experimental capacity data for the estimation of the remaining useful life. The data-driven prognostic approach is highly dependent on the availability of high quantity of quality observations. Insufficient amount of available data can result in unsatisfactory prognostics. In this paper, the prognostics scheme is utilized to estimate the remaining useful life of a battery, with insufficient direct data available, but taking advantage of observations available from a fleet of similar batteries with similar working conditions. Experimental results show that the proposed prognostic scheme provides a fast and efficient estimation of the remaining useful life of the batteries and achieves superior results when compared with various state-of-the-art prediction techniques.

Keywords: Prognostics; extreme learning machines; one-step prediction; multi-steps prediction; Lithium-ion batteries. 


\section{Introduction}

Prognostics of Lithium-ion batteries has become of primary importance in the electronics industry. $-\underline{1}-\underline{3}$ In this industry, prognostics and health management (PHM) techniques are being utilized for improving operation and management, and extending the lifecycle of systems, machines and batteries, decreasing the risk of failures and associated unscheduled maintenance costs. ${ }^{4}$

Quick and accurate estimation of the remaining useful life (RUL) can reduce the failure occurrences of batteries. ${ }^{5,6}$ The RUL is a valuable information for the estimation of a feasible time for battery replacement. The RUL of a battery depends on the battery degradation level and operational conditions. ${ }^{7}$ The nonlinear and uncertain degradation profiles of batteries call for nonlinear predictive models. Monitoring of a battery state during its operational usage has two elements, namely state of charge (SOC) and state of health ( $\mathrm{SOH}) . \stackrel{8}{\mathrm{SOC}}$ is a measure of remaining available stored energy ${ }^{9}$ and $\mathrm{SOH}$ is a measure of the battery degradation. ${ }^{10}$ These measures provide useful information on the present conditions of a battery. $\frac{11}{\text { The }}$ RUL prediction task can, then, be performed through a wide range of prognostic methods, that are mainly classified under two major categories of model-based and data-driven methods. $12-17$

Model-based approaches account for a majority of the modern RUL estimation methods, but often in practice it is difficult to have a precise and well-established model, $\frac{18}{,}$ tune its parameters, and update their values during the prediction phase, for changing operational conditions. ${ }^{19}$ For example, rapid changes at the final cycles of usage increase the difficulty to learn the final steps of the degradation profiles. 7

Data-driven approaches help address these issues in RUL prediction. .5 Datadriven approaches utilize existing and past observations of the batteries degradation profiles for estimating future values. $\frac{19}{}$ RUL can be estimated by learning the relationships amongst the degradation features and the degradation trends. ${ }^{19}$ Informative features, like voltage, capacity, current and impedance have been extracted and used for battery RUL prediction. The numbers of observations and informative features play a vital role for achieving an accurate prediction model. ${ }^{20}$ The data-driven approaches are much faster and easier to implement compared to model-based approaches, ${ }^{21}$ even though they require a large number of observations in the training phase and the prediction model is non-transparent. ${ }^{14}$

The model-based approaches can be classified into two main categories. The first group makes use of the degradation modelling strategy. ${ }^{19}$ The approaches can use a nonlinear degradation model,, 22 an adaptive and nonlinear model,, 22 a hierarchical model to integrate discharging and degradation processes,, 23 a sparse Bayeshian predictive model $^{24}$ and a degraded Markov model ${ }^{14}$ to estimate the remaining use life. Such models are a bit complex and very sensitive to the quality and quantity of the battery data. The second group makes use of the state/parameter observers, like extended Kalman filter ${ }^{25}$ and particle filter. ${ }^{26}$ These approaches are computationally expensive and sensitive to the accuracy and robustness of the model. The data-driven approaches can be classified into three major categories. This group 
of approaches makes use of statistical measures. $\frac{6}{}$ These techniques are quite simple and straightforward. The second group of approaches aim to map from extracted features or stressing factors to state of health. These include the use of support vector machine ${ }^{27,28}$ and neural networks. ${ }^{29,30}$ These techniques can be easily implemented and have a good nonlinear mapping capability. The third group of approaches aim to map directly from aging cycle to state of health. $\frac{31,32}{}$ These techniques have a good nonlinear mapping capability but are difficult to be implemented. All these techniques are sensitive to the quantity and quality of the battery data. Several data-driven approaches, including support vector machines, ${ }^{27,28}$ relevance vector machines, ${ }^{33}$ and artificial neural networks ${ }^{30}$ have been implemented for the prediction of remaining useful life of batteries. ${ }^{34}$ However, many of such techniques suffer from computational complexity and higher computational time. ${ }^{34}$

The work in this paper focuses on the study and comparison of data-driven techniques for the one-step-ahead prediction (OSP) and the multi-step-ahead prediction (MSP) of batteries RUL. The aim is to develop several predictors based on extreme learning machines $(\mathrm{ELM})^{35-\underline{37}}$ for estimation of the remaining useful life of batteries. Extreme learning machines (ELM) are known for fast implementation, low complexity and reliable predictions. The proposed ELM-based predictor is studied and compared to other state-of-the-art prediction techniques, including

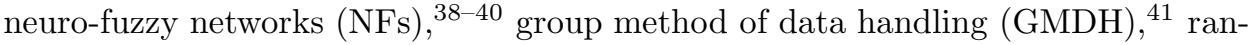
dom forests $(\mathrm{RF})^{42}$ and echo state networks (ESN). ${ }^{43}$ These predictors are formed in the nonlinear autoregressive (NAR) structures for OSP and used along within several MSP structures, namely iterative, direct and DirRec, for the long-term prediction. All these strategies create different training subsets including current and past observations of the batteries and train several models to predict the future capacity values in short and long horizons.

The paper also tries to handle the situation of estimating the RUL of a battery, with insufficient or unusable observations. There is often a situation, particularly in case of a new equipment, that the available set of observations or data lack the quantity as well as the quality to be suitable to generate a training subset. The paper proposes a solution to this problem by utilizing the data available from a fleet of existing batteries. These batteries are similar and operate under similar working conditions and degradation processes. The data from the fleet of batteries are combined to train the prognostic model. Once trained, the model can be utilized to estimate the RUL of the battery.

The remainder of the paper is structured as follows. Section 2 of the paper briefly formulates the problem statement. Section 3 presents the proposed techniques to predict the remaining useful life of the Lithium-ion batteries. Section 4 discusses and analyses the experimental results. Section 5 presents the conclusion.

\section{Problem Statement}

The primary goal of the paper is to analyze the degradation process in Lithium-ion batteries and estimate their remaining useful life over both short and long time 


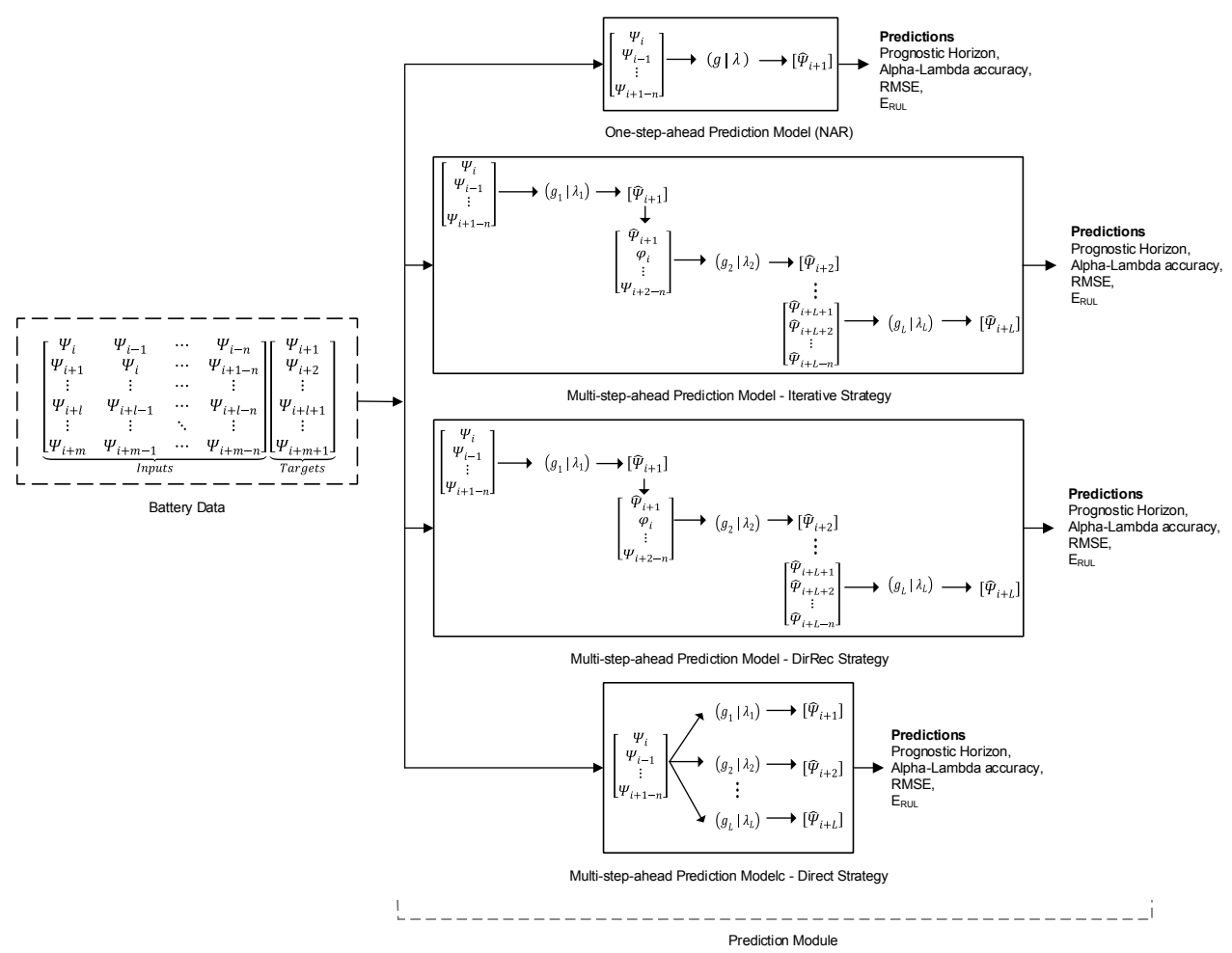

Fig. 1. Block diagram of the prediction system. Battery data are fed into the predictive models for estimating the RUL of the battery.

horizons. Four different battery datasets, namely $B 5, B 6, B 7 B 18$, are used for the estimation of the remaining useful life in terms of capacity. These datasets were collected at the Idaho National Lab and can be obtained from the NASA Prognostic Center of Excellence Data Repository. $\stackrel{44}{*}$ The batteries have been charged at the constant rate of $1.5 \mathrm{~A}$, till the voltage reaches $4.2 \mathrm{~V}$ and, then, the voltage is sustained at a constant level till the current reduces to $20 \mathrm{~mA}$. In discharge cycles, the batteries maintain a constant current level till the voltage drops below a preset value. ${ }^{14}$ Batteries $B 5, B 6, B 7$ and $B 18$ are considered to be fully discharged when their voltage drops to $2.7 \mathrm{~V}, 2.5 \mathrm{~V}, 2.2 \mathrm{~V}$ and $2.5 \mathrm{~V}$ respectively. This charge and discharge cycles are continued until the rated capacity drops from $2 \mathrm{Ahr}$ to $1.4 A h r$, a drop of $30 \%$, which is a criteria for battery end-of-life. ${ }^{21}$ Capacity of a battery, $C(A h r)$, is a common index to predict its remaining useful life ${ }^{45}$ and, hence, in this paper, capacities from 168 discharge cycles are extracted and utilized to create required inputs for the predictors in order to estimate the RUL of the batteries.

Figure 1 shows the proposed prediction scheme. The following prediction strategies can be considered to construct various predictors and estimate the useful life of the Li-ion batteries for short and long-term horizons. 


\subsection{One-step-ahead prediction (OSP) strategy}

One-step-ahead predictions (OSP) are utilized for the estimation of the RUL of a battery over a short horizon. Nonlinear autoregressive (NAR) structures ${ }^{46}$ are used to construct predictive models using the following equation:

$$
\hat{\psi}_{i+1}=g\left(\psi_{i}, \psi_{i-1}, \psi_{i-2}, \ldots, \psi_{i+1-n}\right)+\epsilon_{i+1}
$$

where $\psi$ symbolizes the battery capacity at discharge cycles, $n$ symbolizes the number of lags, $\epsilon$ symbolizes the prediction error for each cycle, $i$ symbolizes the cycle number, and $g$ symbolizes the prediction approximation function during the training phase.

\subsection{Multi-step-ahead prediction (MSP) strategies}

(a) Iterative strategy predicts the next future value using OSP method and, then, uses the predicted value as a known input iteratively to predict the subsequent $L$ future values. Prediction of the $L$ subsequent values using this technique can be formulated by the following equation

$$
\hat{\psi}_{i+l}= \begin{cases}g\left(\psi_{i}, \ldots, \psi_{i+1-n}\right), & \text { if } l=1 \\ g\left(\hat{\psi}_{i+l-1}, \ldots, \hat{\psi}_{i+1}, \psi_{i}, \ldots, \psi_{i+l-n}\right), & \text { if } l \in\{2, \ldots, n\} \\ g\left(\hat{\psi}_{i+l-1}, \ldots, \hat{\psi}_{i+l-n}\right), & \text { if } l \in\{d+1, \ldots, L\}\end{cases}
$$

where $\psi$ symbolizes the battery capacity at discharge cycles, $\hat{\psi}$ symbolizes the predicted battery capacity, $n$ symbolizes the number of lags, $i$ symbolizes the cycle number, $g$ symbolizes the one-step prediction model and $L$ symbolizes the total number of predictions. ${ }^{47,48}$

(b) DirRec strategy predicts multi-step-ahead values similar to iterative strategy, but, generates a new prediction model after each prediction step. ${ }^{48}$ Prediction of $L$ subsequent values using this technique can be formulated by the following equation

$$
\hat{\psi}_{i+l}= \begin{cases}g_{l}\left(\psi_{i}, \ldots, \psi_{i+1-n}\right), & \text { if } l=1 \\ g_{l}\left(\hat{\psi}_{i+l-1}, \ldots, \hat{\psi}_{i+1}, \psi_{i}, \ldots, \psi_{i+l-n}\right), & \text { if } l \in\{2, \ldots, n\} \\ g_{l}\left(\hat{\psi}_{i+l-1}, \ldots, \hat{\psi}_{i+l-n}\right), & \text { if } l \in\{n+1, \ldots, L\}\end{cases}
$$

where $g_{l}$ symbolizes the predictive model constructed at the $l$ th prediction step. $\underline{49}$

(c) Direct strategy constructs $L$ distinct models $g_{l}$ to predict $L$ future values using the following equation $\frac{49}{}$

$$
\hat{\psi}_{i+l}=g_{l}\left(\psi_{i}, \psi_{i-1}, \ldots, \psi_{i+1-n}\right), \quad l \in[1, L] .
$$




\subsection{Fleet-based predictions}

The one-step ahead predictive model for the fleet-based prognostic scheme is generated using an ensemble of one-step ahead prediction models for all available batteries. For the iterative multi-step ahead predictions, the first future value is estimated using the one-step model, which is then used as known input iteratively to generate subsequent future values

$$
\hat{\psi}_{k, i+1}=g_{\text {fleet }}\left(\psi_{k, i}, \psi_{k, i-1}, \psi_{k, i-2}, \ldots, \psi_{k, i+1-n}\right)
$$

where $k$ symbolizes the $k$ th battery in the available fleet and $g_{\text {fleet }}$ symbolizes the one-step ahead predictive models for the various batteries in the fleet.

\section{Predictor Models}

\subsection{Extreme Learning Machine (ELM)}

ELM is a generalized, single hidden layer feedforward network (SLFN), $\frac{35,36}{2}$ with the following output function 50

$$
f_{p}(\mathrm{x})=\sum_{i=1}^{p} \beta_{i} h_{i}(\mathrm{x})=\mathrm{h}(\mathrm{x}) \beta, \quad x \in \mathbb{R}^{n}, \quad \beta_{i} \in \mathbb{R}^{o}
$$

where $p$ is the number of hidden nodes, $\beta=\left[\beta_{1}, \ldots, \beta_{p}\right]^{T}$ stands for the vector of output weights linking nodes of the hidden layer to the $o \geq 1$ output nodes, $h(x)=\left[h_{1}(x), \ldots, h_{p}(x)\right]^{T}$ denotes the ELM nonlinear feature mapping. Hidden nodes may include different output functions. Particularly, $h_{i}(x)$ can be defined as

$$
h_{i}(x)=f\left(\omega_{i} x+b_{i}\right), \quad \omega_{i} \in \mathbb{R}^{p}, \quad b_{i} \in \mathbb{R}^{p}
$$

where $f(\omega x+b)$ stands for a nonlinear piecewise continuous function, which satisfies the universal approximation capability of ELM, in which $(\omega, b)$ are the hidden node parameters. $\frac{35}{}$

Given a set of observations $\mathbb{S}=\left\{\left(x_{j}, y_{j}\right) \mid x_{j} \in \mathbb{R}^{n}, y_{j} \in \mathbb{R}^{o}\right\}$, a standard SLFN with $p$ hidden nodes and an arbitrary activation function can be modeled as follows

$$
f_{p}\left(x_{j}\right)=\sum_{i=1}^{p} \beta_{i} h_{i}\left(x_{j}\right)=\sum_{i=1}^{p} \beta_{i} f\left(\omega_{i} x_{j}+b_{i}\right), \quad j=1, \ldots, m
$$

where $\omega_{i}=\left[\omega_{i 1}, \ldots, \omega_{i p}\right]^{T}$ stands for the weight vector linking the $i$ th hidden node and the input nodes, $b_{i}$ denotes the threshold of the $i$ th hidden node and $f$ stands for the activation function. There exist various types of activation functions such as sigmoid, hyperbolic tangent, Gaussian, hard limit, cosine/Fourier basis, sine and radial basis function (RBF). ${ }^{35}$ Here, the sigmoid function has been used, which can be defined as follows

$$
f\left(\omega_{i} x_{j}+b_{i}\right)=\frac{1}{1+\exp \left(-\left(\omega_{i} x_{j}+b_{i}\right)\right)}, \quad \omega_{i} \in \mathbb{R}^{p}, \quad b_{i} \in \mathbb{R}^{p} .
$$


ELM randomly generates $p$ hidden nodes (step 1), without the need for tuning, to approximate the input-output relation among the $m$ pairs of observations with the minimum training error, $\varepsilon_{j}=\sum_{i=1}^{p}\left\|f_{p}\left(x_{j}\right)-y_{j}\right\|$, i.e., it determines $\left(\omega_{i}, b_{i}\right)$ and $\beta_{i}$ such to satisfy the following

$$
\sum_{i=1}^{p} \beta_{i} f\left(\omega_{i} x_{j}+b_{i}\right)=y_{j}+\varepsilon_{j}, \quad j=1, \ldots, m
$$

where $\varepsilon$ stands for the error or noise and $y_{j}$ denotes the output vector at the $j$ th row. This can be reformulated as follows

$$
H \beta=Y
$$

where

$$
\begin{gathered}
H=\left[\begin{array}{c}
h\left(x_{1}\right) \\
\vdots \\
h\left(x_{m}\right)
\end{array}\right]=\left[\begin{array}{ccc}
f\left(\omega_{1} x_{1}+b_{1}\right) & \cdots & f\left(\omega_{p} x_{1}+b_{p}\right) \\
\vdots & \ddots & \vdots \\
f\left(\omega_{1} x_{m}+b_{1}\right) & \cdots & f\left(\omega_{p} x_{m}+b_{p}\right)
\end{array}\right]_{m \times p} \\
\beta=\left[\begin{array}{c}
\beta_{1}^{T} \\
\vdots \\
\beta_{p}^{T}
\end{array}\right]_{p \times o}
\end{gathered}
$$

and

$$
Y=\left[\begin{array}{c}
y_{1}^{T} \\
\vdots \\
y_{m}^{T}
\end{array}\right]=\left[\begin{array}{ccc}
y_{11} & \ldots & y_{1 o} \\
\vdots & \ddots & \vdots \\
y_{m 1} & \ldots & y_{m o}
\end{array}\right]_{m \times o}
$$

in which $H$ stands for the randomized matrix of the hidden layer output of the network and $Y$ stands for the target matrix. ELM computes the matrix of hidden layer outputs $H$ (step 2) and, then, computes the vector of output weights (step 3).

The optimal solution can be found, then, by the smallest least-square solution of the linear system (see Eq. (11)) as follows

$$
\hat{\beta}=H^{\dagger} Y
$$

where $H^{\dagger}$ stands for the Moore-Penrose generalized inverse of matrix $H .^{35}$

Figure 2 illustrates the ELM training structure, where the output matrix is generated using the input matrix and the hidden layer matrix. The hidden layer parameters can be generated randomly, independent of the input matrix and, thus, decrease considerably the training time of the model. 


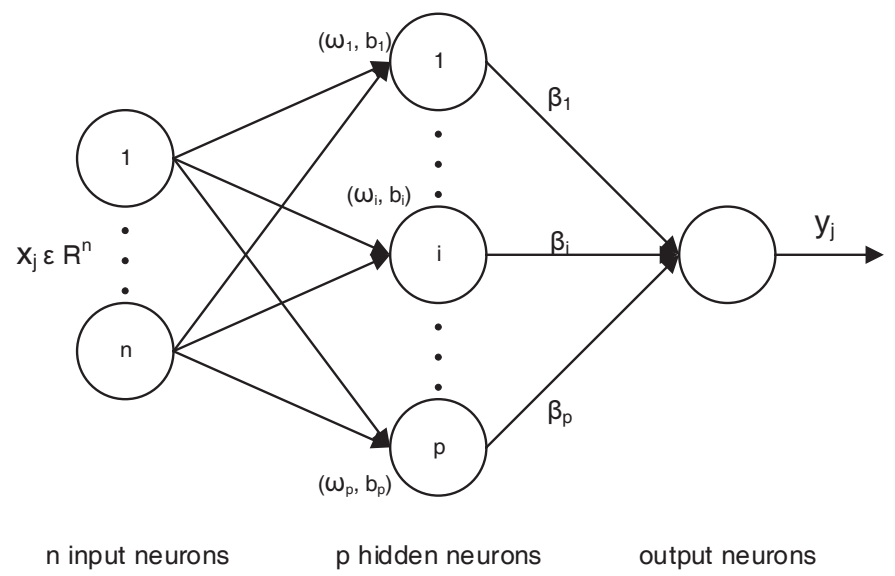

Fig. 2. Extreme learning machine model topography.

\section{Experimental Results}

The proposed prognostic scheme uses the ELM predictor and other state-of-theart methods, including GMDH, NF, RF and ESN, in a set of experiments for one-step and multi-steps ahead predictions. Results are compared in terms of root mean square error (RMSE), prediction error $\left(E_{R U L}\right)$, prognostic horizon and alphalambda accuracy. RMSE calculates the difference between the target and predicted values. $E_{R U L}$ indicates the error in the prediction at the end of life that can be calculated as the difference between the actual and predicted cycle numbers at which the battery reaches the end of life, when the value of capacity crosses a predetermined threshold. For these experiments, the threshold values for batteries $B 5, B 6$ and $B 7$ are $1.299 \mathrm{~V}, 1.424 \mathrm{~V}$ and $1.41 \mathrm{~V}$, respectively. Prognostic horizon indicates the difference of the battery end of life cycle and the cycle where the prediction first reaches a specified performance criteria. .51 For these experiments, the alpha for the prognostic horizon is set at $10 \%$. Alpha-lambda accuracy indicates the quality of estimations by checking if the estimations are within a specified range at particular cycles. $\frac{51}{}$ For these experiments, the alpha for the accuracy is set at $20 \%$. The fleet-based prognostics scheme utilizes the combined datasets of batteries $B 5, B 6$ and $B 7$ to form the training subset in a set of experiments for one-step and multi-steps ahead predictions of the battery $B 18$. The threshold value for the battery $B 18$ is set at $1.4 \mathrm{~V}$. Only iterative strategy is used for the multi-steps ahead predictions using the fleet-based prognostics.

\subsection{One-step ahead predictions}

Various one-step ahead predictions are performed on all battery datasets by varying the number of input lags from two to five. Besides, the size of the training set is also varied between $40 \%, 60 \%$ and $80 \%$ of the total set. 


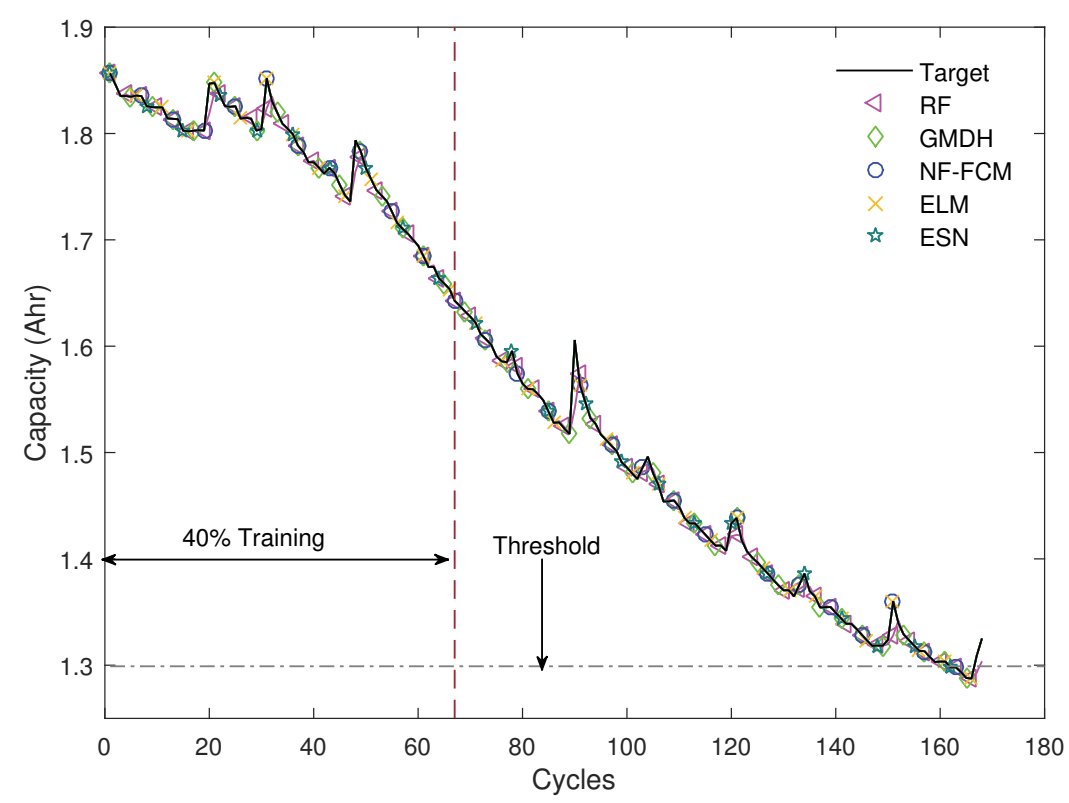

Fig. 3. Prediction results for the test with two input lags and the use of first $40 \%$ of the observations for training.

Figure 3 depicts the prediction result generated by each predictor along with the target for the test $40 \%-2$ on the B5 data. This indicates that the first $40 \%$ of the observations are used for training and the input contains two lags. This figure shows that all methods perform well in predicting the target over a one-step prediction model, since sufficient samples are available for training.

Our experiments indicate that increasing the number of lags does not improve the performance of the predictors.

Figure 4 depicts the distribution of the RMSE for each OS predictor for different tests on all the battery datasets. The boxes represent the distribution range of the RMSE values (dots) between the first and third quartiles, the solid line represents the median value of the RMSE for each OS predictor, the dash lines show the outlier range, the crosses represent the outliers and the solid square represents the mean of the RMSE values for each OS predictor. Presence of the solid line in the middle of the box indicates that the values are uniformly distributed. The predictors are ranked w.r.t. the mean values as ELM, ESN, NF, RF and GMDH. ELM has the smallest box, which indicates the least variation among the RMSE values and the most stable predictions. GMDH produces the biggest window and also has the maximum number of outliers, i.e., the most unstable results.

Figure $\underline{5}$ depicts the distribution of $E_{R U L}$ values for each OS predictor for different tests on all the batteries' datasets. A negative (positive) value for $E_{R U L}$ indicates an early (late) prediction, which means that the prediction profile passes through the threshold sooner (later) than the actual target profile in terms of cycles. 
R. Razavi-Far et al.

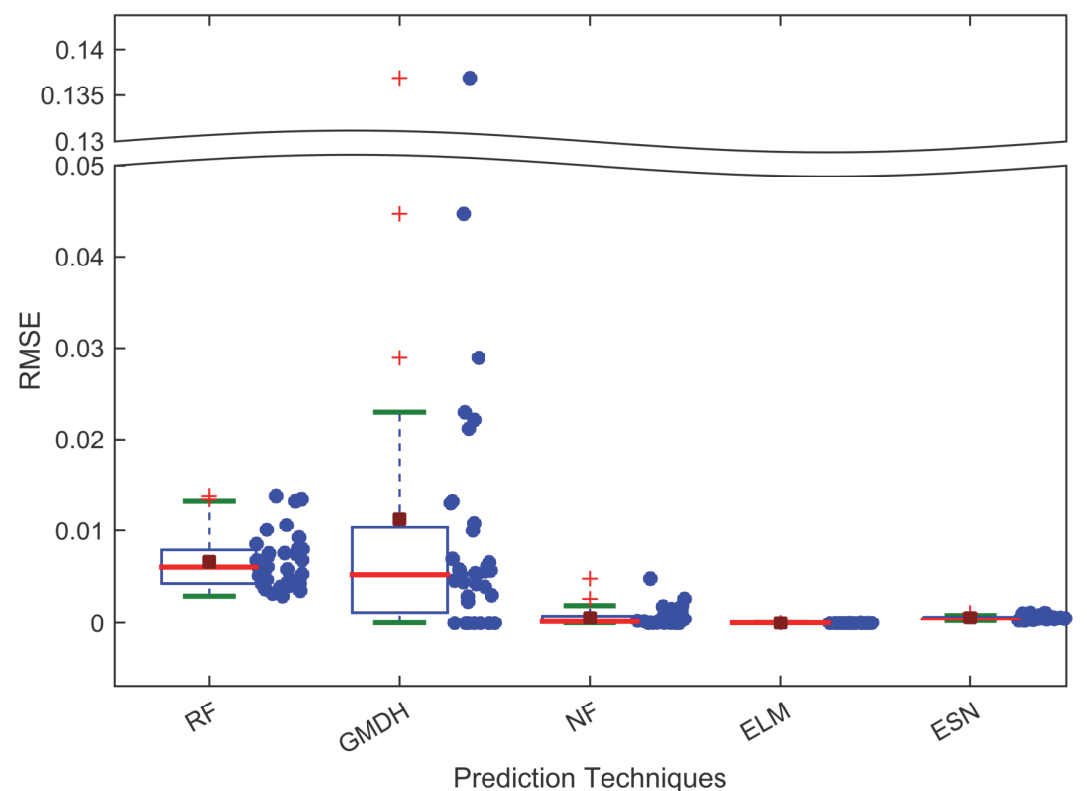

Fig. 4. Box-plots representing the distribution of the RMSE values calculated by each OS predictor, for all performed tests on all battery datasets.

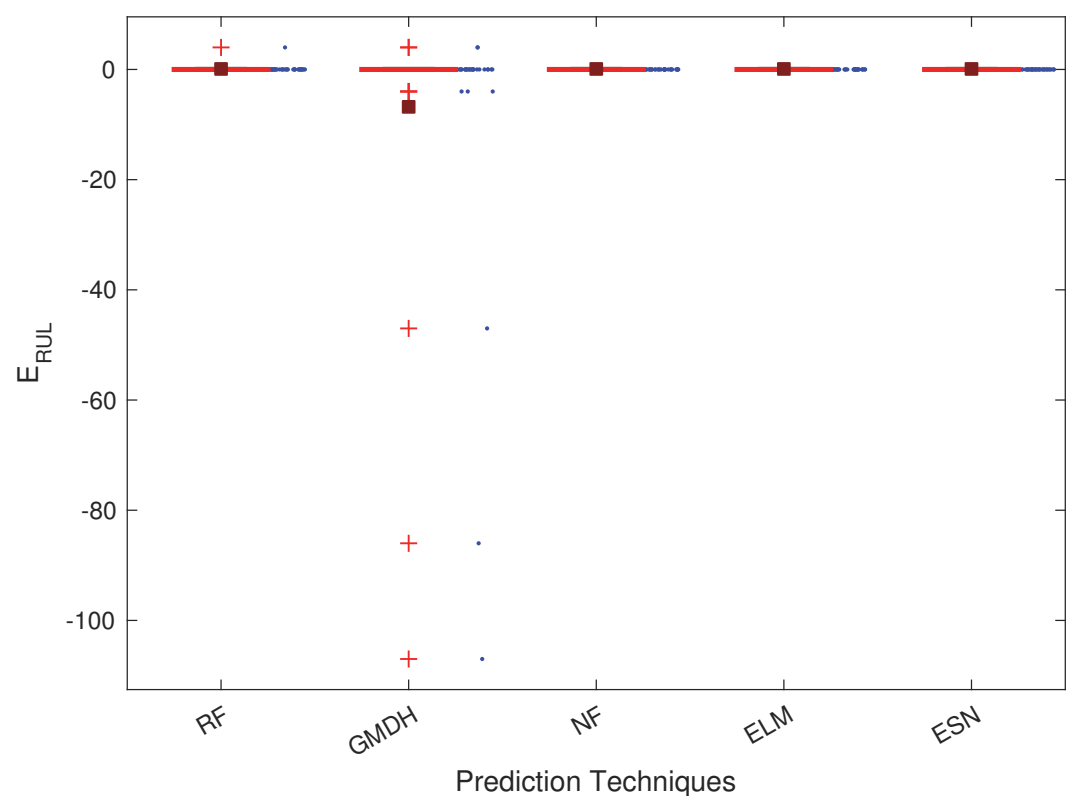

Fig. 5. Box-plots representing the distribution of $E_{R U L}$ values calculated by each OS predictor, for all performed tests on all battery datasets. 
This figure indicates that ELM and NF accurately predict the end of lifecycle. GMDH has several early predictions. The predictors are ranked in terms of $E_{R U L}$ as ELM, ESN, NF, RF and GMDH.

The results for the OS predictions show that ELM is the best OS predictor in terms of RMSE and $E_{R U L}$, and, thus, it can be selected for OS prediction of the RUL of the batteries.

\subsection{Multi-steps ahead predictions}

Various prediction techniques along with the MS prediction strategies (iterative, DirRec and direct) are used to estimate the RUL of batteries over long horizons.

Figure 6 illustrates the MSP results generated by each predictor for all three MSP strategies, along with the target for the test $40 \%-3$ on the B5 data.

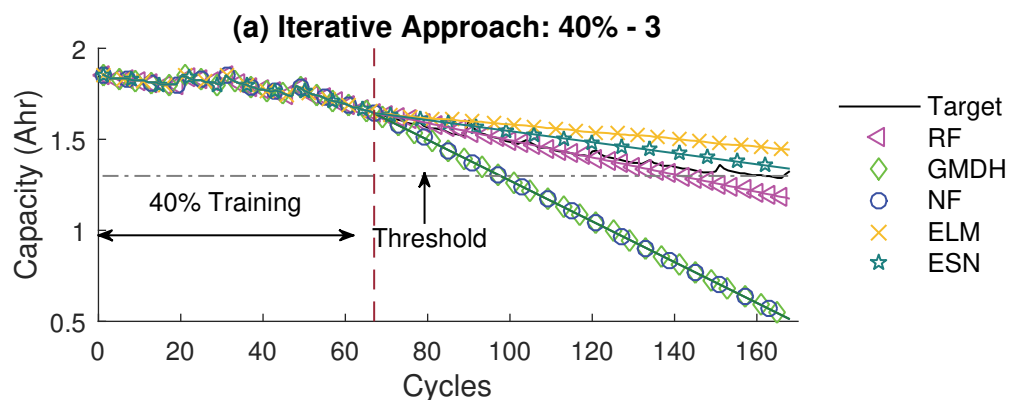

(b) DirRec Approach: $40 \%$ - 3

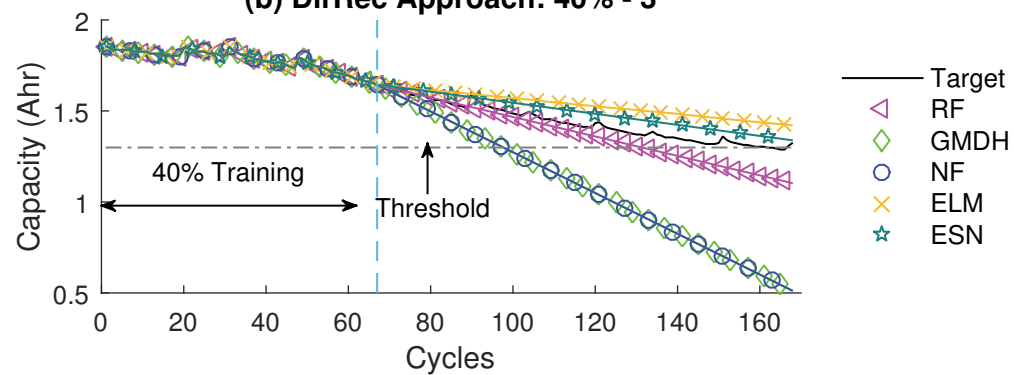

(c) Direct Approach: 40\% - 3

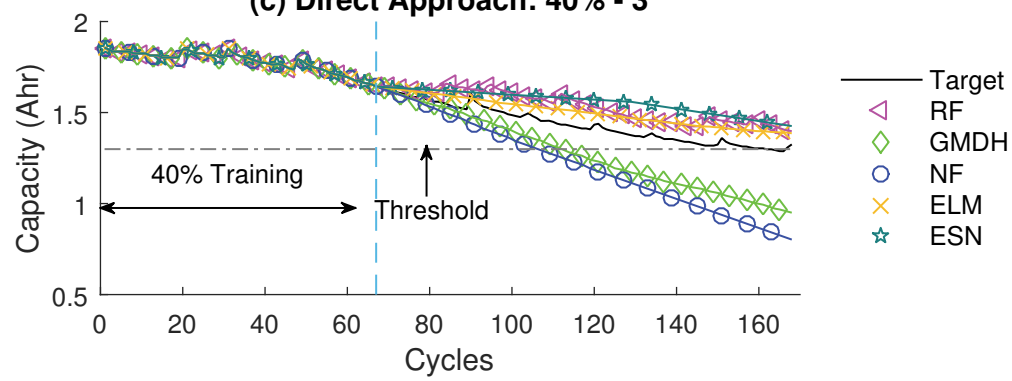

Fig. 6. Prediction results for three strategies using three input lags and the first $40 \%$ of the observations for training. 
This indicates that the input has three lags and the first $40 \%$ of the observations are used for training. Panels (a) and (b) in the figure show that the iterative and DirRec strategies achieve large prediction errors for the small sequence of observations. The error generated at each prediction step propagates through the next steps and leads to a significant deviation from the target. The direct strategy, on the other hand, attains better prediction results for small sequences of observations (see panel c). However, many of the predictors fail to quickly reach the threshold.

Figure 7 illustrates the MSP result generated by each predictor for all three MSP strategies, along with the target for the test $60 \%-3$ on the B5 data. This indicates that the input has three lags and the first $60 \%$ of the observations are used for training. Increasing the length of the sequence of observations improves

(a) Iterative Approach: $60 \%$ - 3
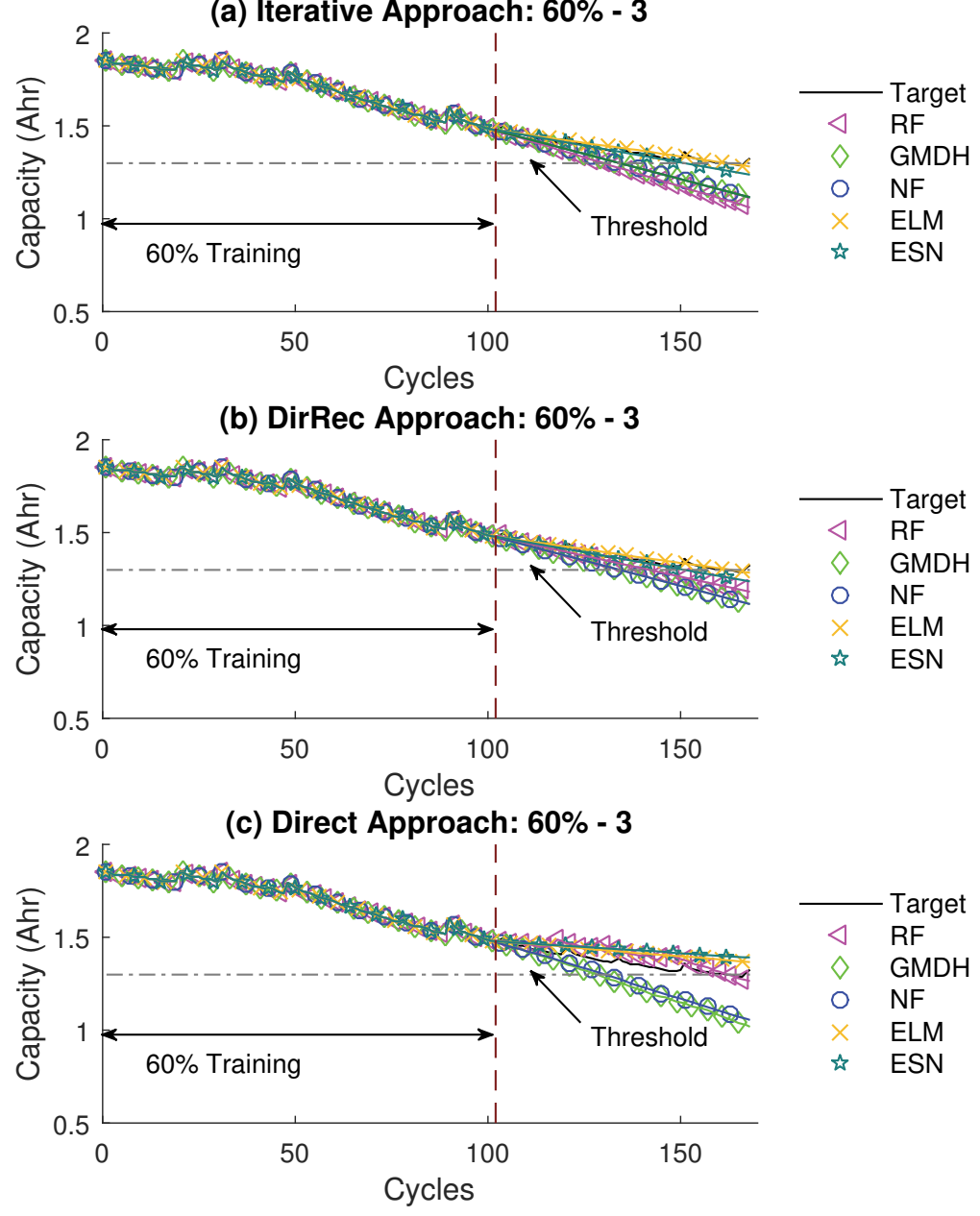

Fig. 7. Prediction results for three strategies using three input lags and the first $60 \%$ of the observations for training. 
(a) Iterative Approach

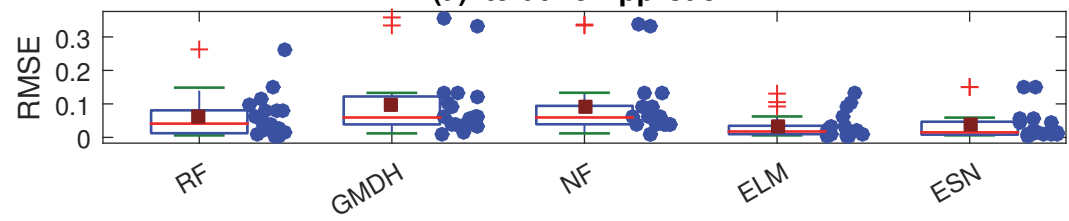

(b) DirRec Approach

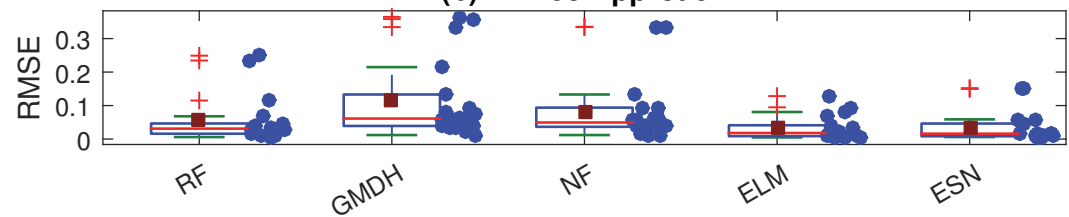

(c) Direct Approach

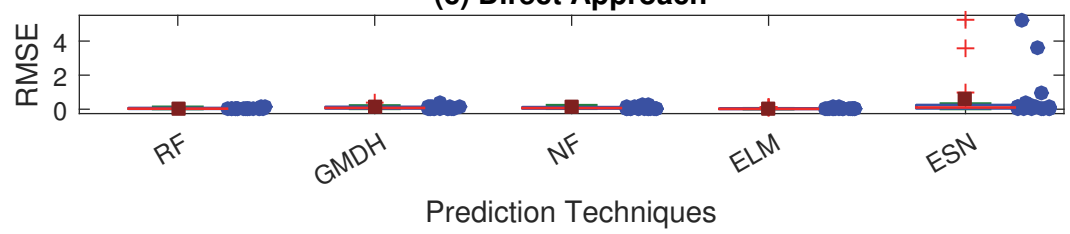

Fig. 8. Box-plots representing the distribution of the RMSE values calculated by each MS predictor on all performed tests for all three strategies.

the prediction result for all three MSP strategies, especially for the iterative and DirRec strategies.

Figure 8 illustrates the distribution of the RMSE for each MS predictor for all three strategies on all the battery datasets. Figure 8 shows that ELM outperforms all predictors and has the lowest mean, the smallest box and thus, the lowest range of outliers. It also shows that the KELM predictors closely follow the ELM predictor. These MS predictors are ranked w.r.t. RMSE as ELM, ESN, RF, NF and GMDH.

Figure 9 illustrates the distribution of the $E_{R U L}$ values for each MS predictor on all the battery datasets. Figure 9 clearly illustrates that ELM outperforms other predictors in estimating the remaining life for all the batteries and has a much smaller box representing more stable results for all MSP strategies. The predictors are ranked w.r.t. to $E_{R U L}$ as ELM, RF, ESN, NF, GMDH.

The attained results can also be studied in terms of multi-steps ahead prediction strategies, which are not presented here due to space limitation. The rank of these strategies, when the predictions generated by the strategies are compared in terms of RMSE and $E_{R U L}$, is direct, DirRec and iterative.

Figure 10 illustrates the prognostic horizon and alpha-lambda accuracy plots for the distribution of $E_{R U L}$ values attained by each MS predictor on all the battery datasets for the iterative strategy. The prognostic horizon plots show that ELM, closely followed by ESN, generate predictions which are always within the boundaries of the prognostic horizon for all battery datasets. In the alpha-lambda accuracy plots, ELM makes the earliest estimation within the accuracy level for 
(a) Iterative Approach

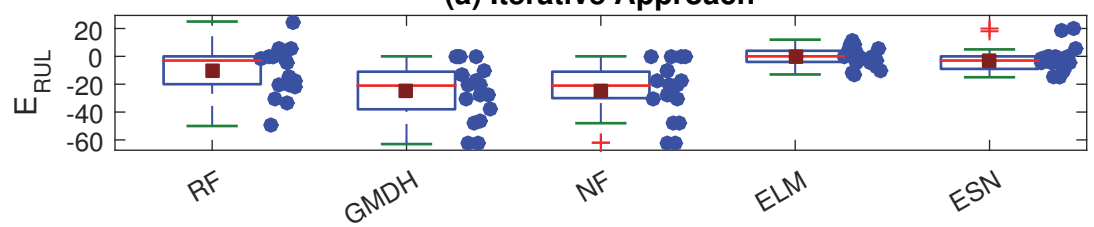

(b) DirRec Approach

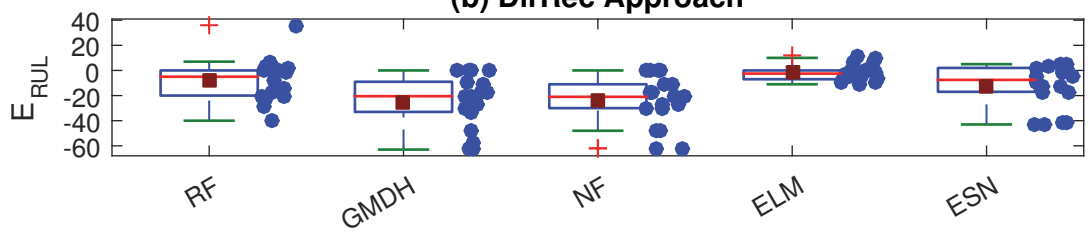

(c) Direct Approach

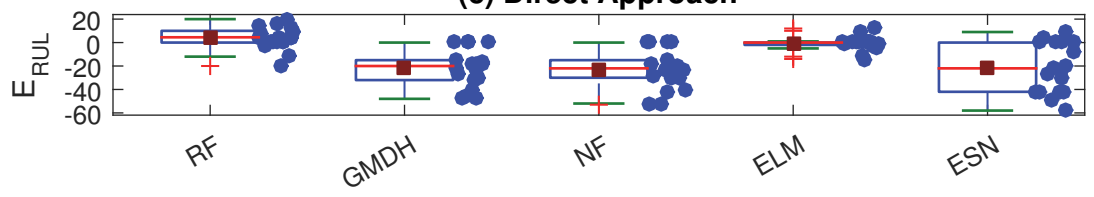

Prediction Techniques

Fig. 9. Box-plots representing the distribution of the $E_{R U L}$ values attained by each MS predictor on all performed tests for all three strategies.

battery datasets and the generated predictions outnumber all other competitors to fall within the accuracy boundaries. The figure shows that ELM can generate accurate and reliable predictions, even with a smaller training subset.

The attained results for the MS predictions show that ELM is the best MS predictor in terms of RMSE, $E_{R U L}$, prognostic horizon and alpha-lambda accuracy and thus, it can be selected for MS prediction of the RUL of batteries.

\subsection{Fleet-based prognostics}

For the purpose of fleet-based prognostics, the combined datasets of the batteries $B 5, B 6$ and $B 7$ are used to generate an overall training subset, which is then used to estimate the RUL of the battery dataset $B 18$. Both OS and MS predictions are performed on the battery dataset $B 18$. Only iterative strategy has been used for the MS predictions, as other techniques are either not suited for a fleet-based prognostics or computationally challenging.

Figure 11 illustrates the OS prediction result generated by each predictor along with the target on the $B 18$ data. This indicates that the entire $100 \%$ of the observations from B5, B6 and B7 are used for training and the input contains five lags. This figure shows that the ELM produces predictions close to the target. Both GMDH and RF show spikes in the predictions, instead.

Figure 12 illustrates the distribution of the RMSE and $E_{R U L}$ values calculated by each OS predictor for fleet-based prognostics. The figure shows that ELM pro- 

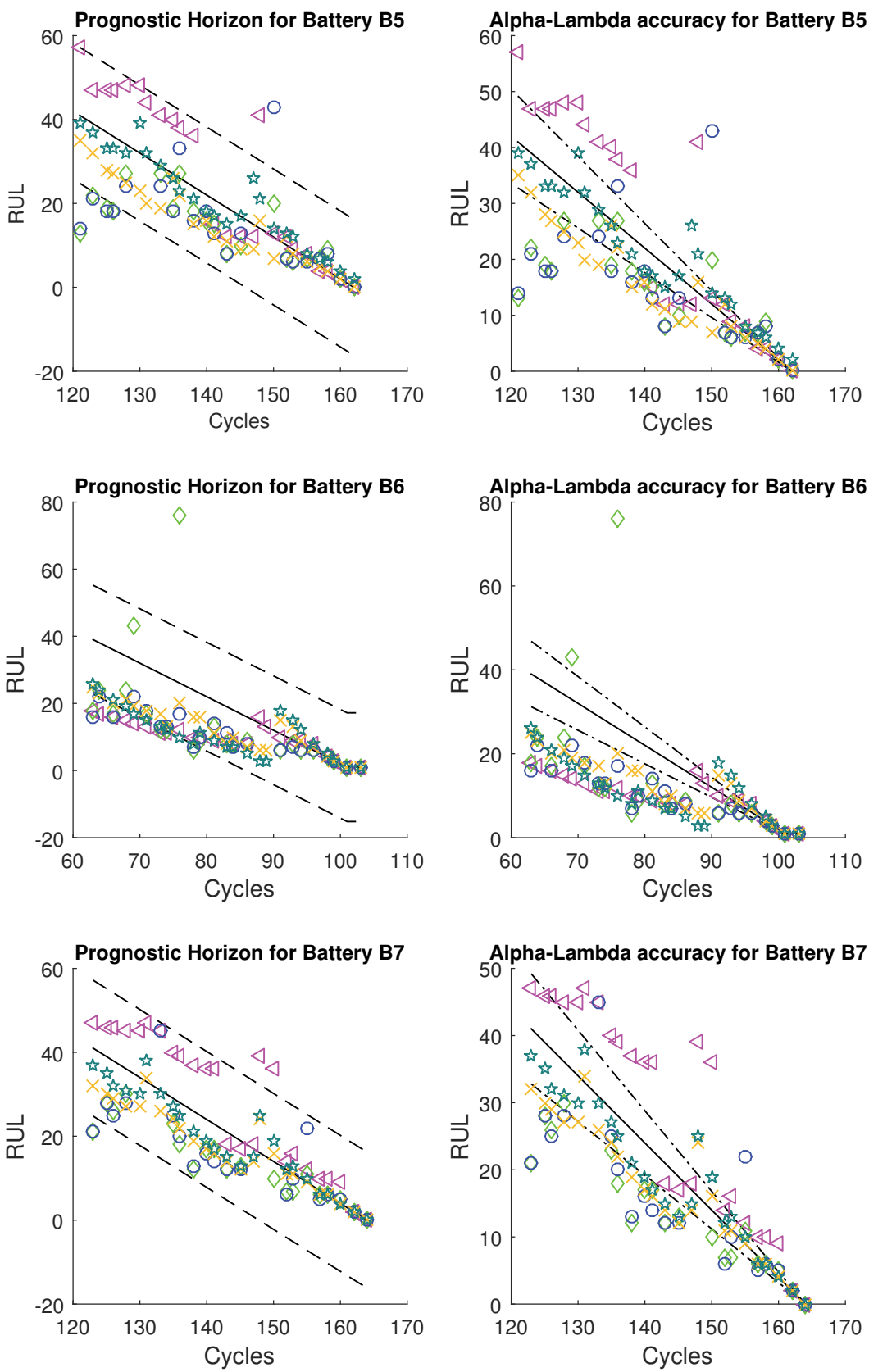

Fig. 10. Prognostic horizon and alpha-lambda accuracy plot representing the distribution of the $E_{R U L}$ values attained by each MS predictor on all performed tests for iterative strategy. 


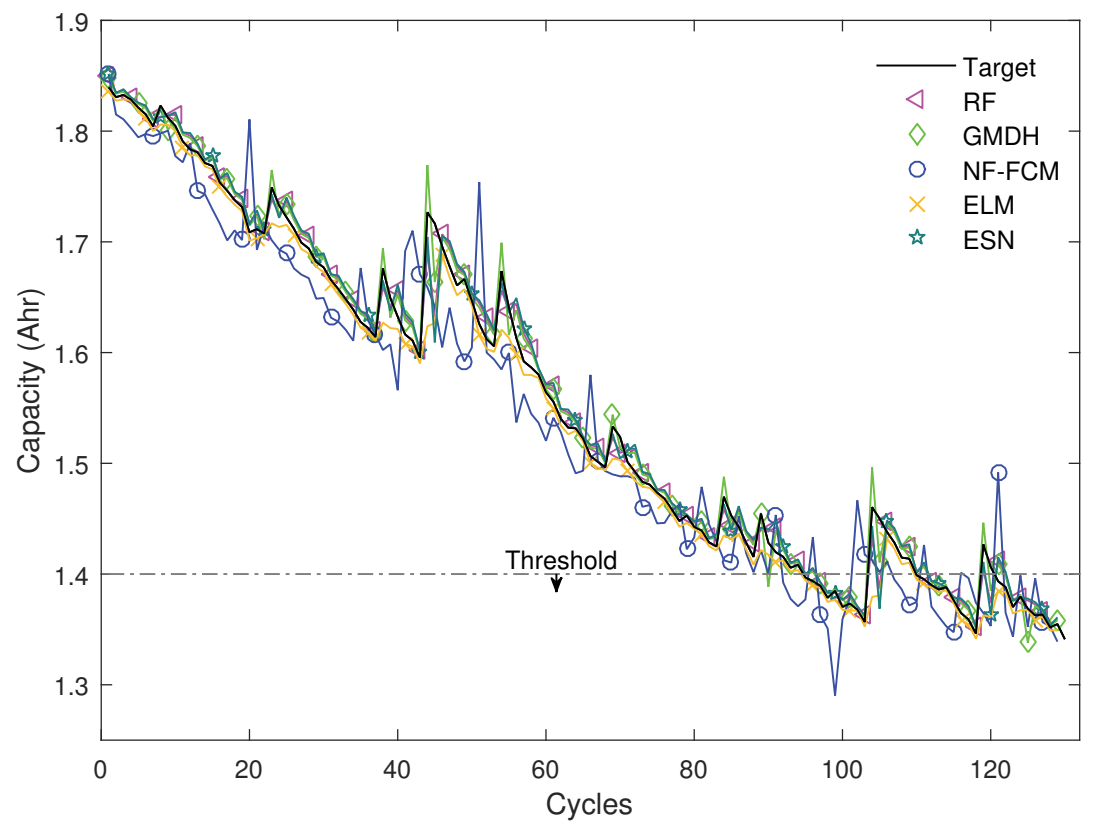

Fig. 11. OS prediction results for battery $B 18$ using three input lags and the entire observations of the three batteries for training.
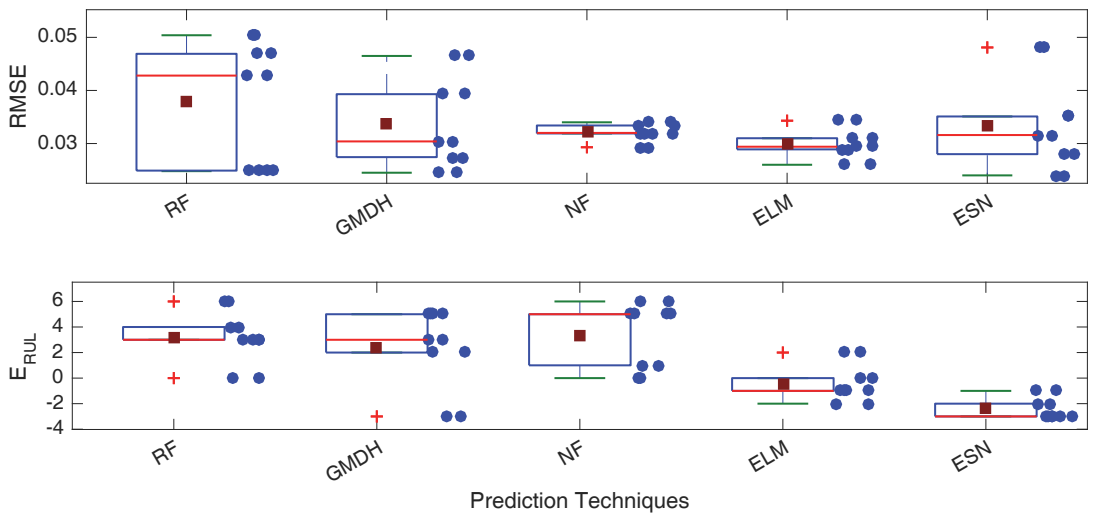

Fig. 12. Box-plots representing the distribution of the RMSE and $E_{R U L}$ values calculated by each OS predictor for fleet-based prognostics.

duces lower RMSE, accurate RUL predictions and more stable results, indicated by the smaller box size. The predictors are ranked w.r.t. to RMSE as ELM, NF, ESN, GMDH and RF and w.r.t $E_{R U L}$ as ELM, ESN, GMDH, RF and NF.

Figure 13 illustrates the MS prediction result generated by each predictor along with the target on the $B 18$ data using the iterative strategy. This indicates that the entire $100 \%$ of the observations from B5, B6 and B7 are used for training and the 
Extreme Learning Machine Based Prognostics of Battery Life

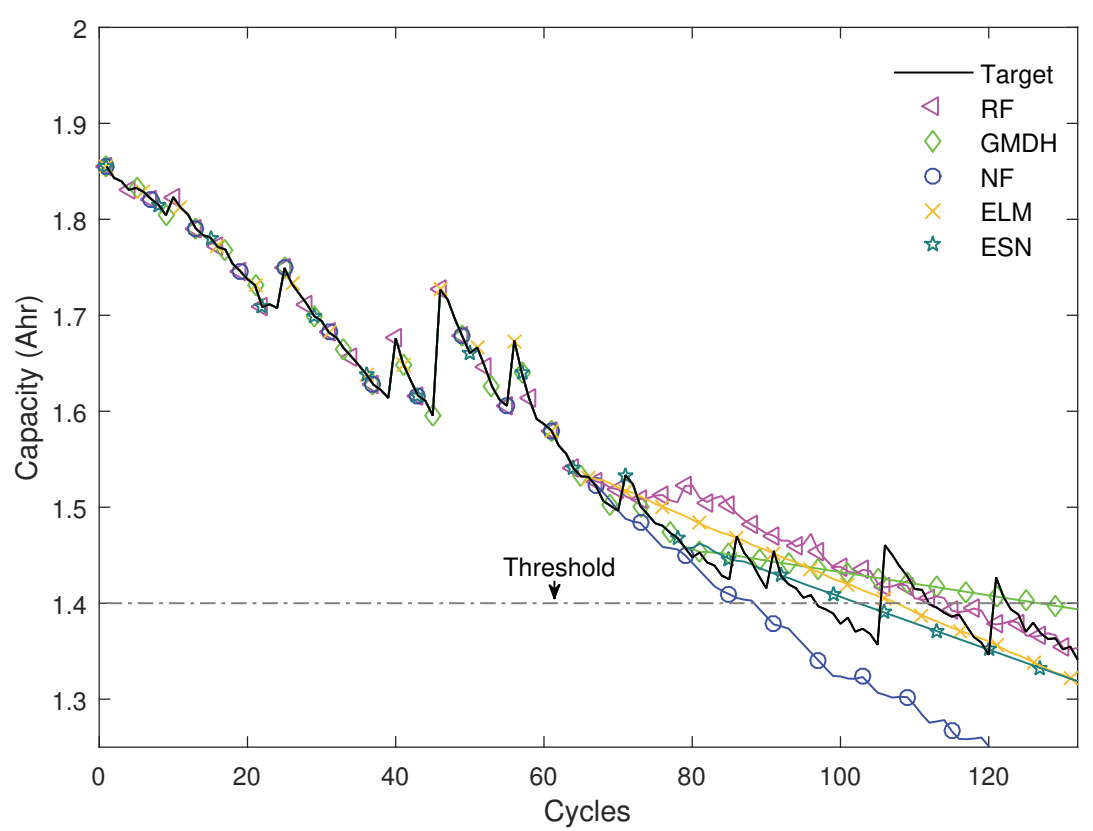

Fig. 13. MS prediction results for battery $B 18$ using iterative strategy, three input lags and the entire observations of the three batteries for training.

input contains three lags. This figure shows that all predictors, except NF, produce predictions close to the target.

Figure 14 illustrates the distribution of the RMSE and $E_{R U L}$ values calculated by each MS predictor for fleet-based prognostics. The figure shows that, similar to OS predictions, ELM produces lower RMSE, accurate RUL predictions and more stable results, indicated by the smaller box size. The predictors are ranked w.r.t.
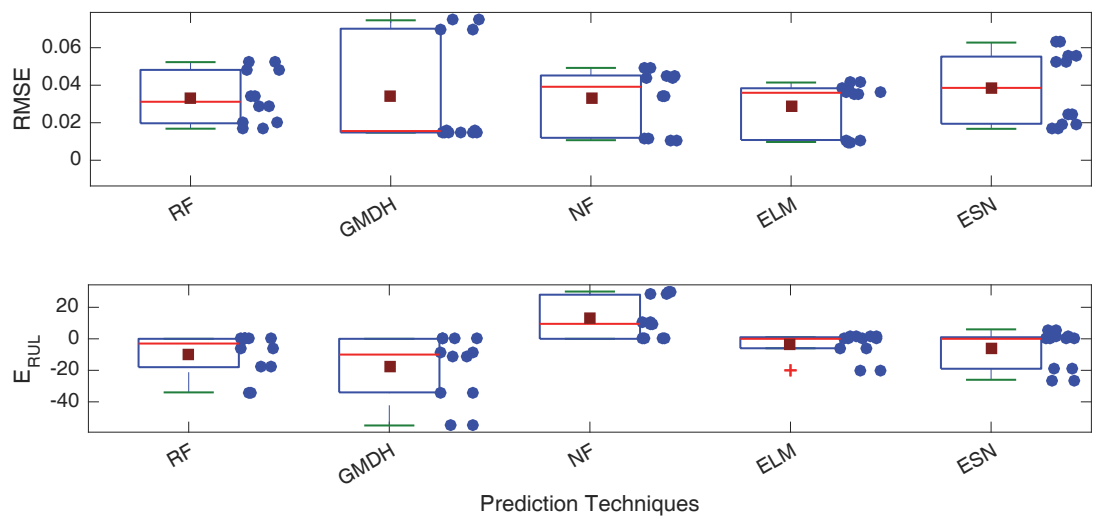

Fig. 14. Box-plots representing the distribution of the RMSE and $E_{R U L}$ values calculated by each MS predictor for fleet-based prognostics. 

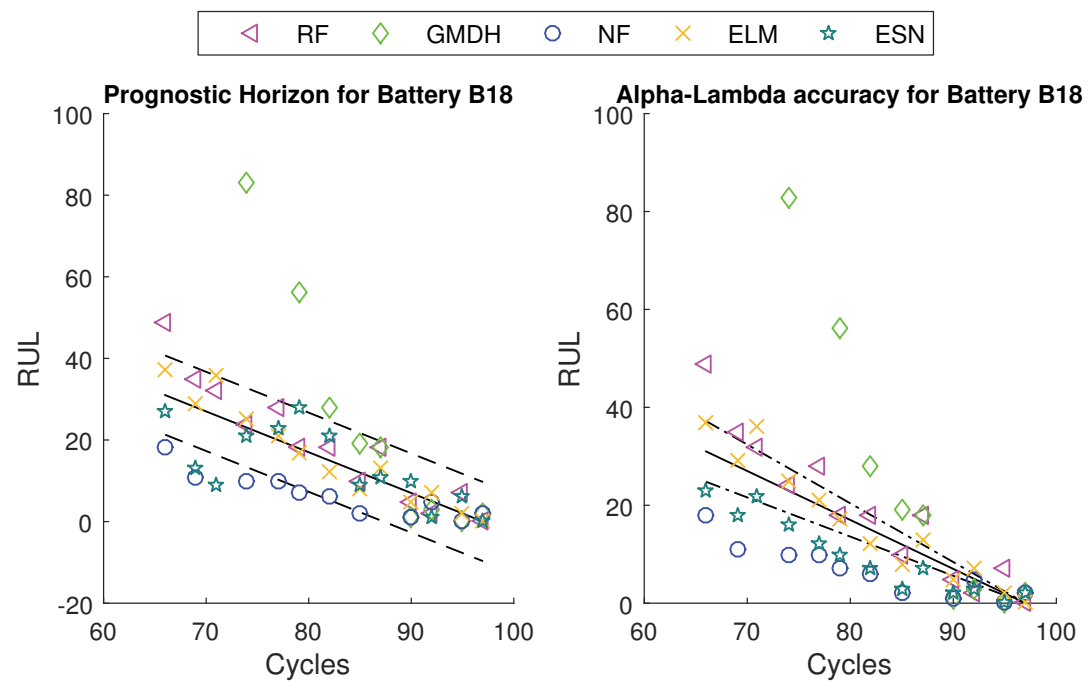

Fig. 15. Prognostic horizon and alpha-lambda accuracy plot representing the distribution of the $E_{R U L}$ values attained by each MS predictor for iterative strategy.

to RMSE as ELM, NF, ESN, GMDH and RF and w.r.t. $E_{R U L}$ as ELM, ESN, RF, GMDH and NF.

Figure 15 illustrates the prognostic horizon and alpha-lambda accuracy plots for the distribution of $E_{R U L}$ values attained by each MS predictor on the battery $B 18$ dataset for the iterative strategy. The prognostic horizon plots clearly show that ELM is the only predictor whose predictions are always within the boundaries of the prognostic horizon for all battery datasets. In the alpha-lambda accuracy plots, ELM makes the earliest predictions within the accuracy level for battery datasets and on aggregate, predicts EOL within the accuracy boundaries more than other predictors. This indicates that ELM is an excellent predictor for fleet-based prognostics, producing accurate and reliable predictions.

\section{Conclusions}

This paper proposes an efficient prognostic scheme for one-step and multi-step ahead predictions of the remaining useful life of Lithium-ion batteries. An Extreme Learning Machine-based prediction technique has been devised along with various one-step and multi-step prediction strategies to estimate the RUL of Lithium-ion batteries. The primary goal of the paper is to deliver a fast and efficient datadriven prognostic scheme to predict the remaining life of batteries over short and long time horizons, even when insufficient observations are available. The attained results show that ELM prediction techniques are fast when compared to other competitors and generate accurate prediction profiles. The ELM predictive model outperforms other competitors for the one-step ahead and the multi-step ahead 
RUL predictions. The ELM predictive model also outperforms the competitors for the fleet-based prognostics.

The implementation of an effective prediction model can often be challenging. The performance of data driven prediction models highly depend on the availability of observations. However, excessive amount of information can often lead to overfitting of data. Observations for a time-series data, similar to the ones used in this paper, often generates different predictive models for different time intervals. Hence, using the entire set of observations as a training dataset may not be ideal to generate a prediction model. The proposed scheme in the paper is designed for a prediction model in a standard batch environment. It could be easily transformed into an online just-in-time environment using online variants of the ELM algorithm. An online prediction model for estimating the remaining useful life of the battery, where new observations are fed into the system, one at a time or in batches, would be an interesting direction for the future research in order to improve the prognostics of battery life.

\section{References}

1. J. Zhang and J. Lee, A review on prognostics and health monitoring of li-ion battery, Jour. of Power Sources 196 (2011) 6007-6014.

2. K. Goebel, B. Saha, A. Saxena, J. R. Celaya and J. P. Christophersen, Prognostics in battery health management, IEEE Instrum. Meas. Mag. 8 (2008) 33-40.

3. T. Benkedjouhb, K. Medjahera, N. Zerhounia and S. Rechakc, Remaining useful life estimation based on nonlinear feature reduction and support vector regression, Engineering Applications of Artificial Intelligence 26(7) (2013) 1751-1760.

4. Y. Zhang, G. W. Gantt, M. J. Rychlinski, R. M. Edwards, J. J. Correia and C. E. Wolf, Connected vehicle diagnostics and prognostics, concept, and initial practice, IEEE Transactions on Reliability 58(2) (2009) 286-294.

5. G. Vachtsevanos, F. Lewis, M. Roemer, A. Hess and B. Wu, Intelligent Fault Diagnosis and Prognosis for Engineering Systems (John Wiley \& Sons, Inc., Hoboken, NJ, USA, 2006).

6. A. Widodo, M.-C. Shim, W. Caesarendra and B.-S. Yang, Intelligent prognostics for battery health monitoring based on sample entropy, Expert Systems with Applications 38(9) (2011) 11763 - 11769.

7. Z. Ye, L. Zhao, Z. Wang, P. Ma, X. Su, L. Pang and M. Pecht, A dual-level approach for lithium-ion battery RUL prognosis, in IEEE Conf. Prognostics $\& 3$ Health Management (Beijing, China, 2015), pp. 1-6.

8. D. Liu, J. Pang, J. Zhou, Y. Peng and M. Pecht, Prognostics for state of health estimation of lithium-ion batteries based on combination Gaussian process functional regression, Microelectronics Reliability 53 (2013) 832-839.

9. S. Malkhandi, Fuzzy logic-based learning system and estimation of state-of-charge of lead-acid battery, Engineering Applications of Artificial Intelligence 19 (2006) $479-485$.

10. K. Chao and J. Chen, State-of-health estimator based-on extension theory with a learning mechanism for lead-acid batteries, Expert Systems with Applications $\mathbf{3 8}$ (2011) 15183-15193. 
11. D. E. Acuna, M. E. Orchard, J. F. Silva and A. Perez, Multiple-imputation-particlefiltering for uncertainty characterization in battery state-of-charge estimation problems with missing measurement data: Performance analysis and impact on prognostic algorithms, Int. J. of Prognostics 85 Health Management 8 (2015) 1-12.

12. L. Liao and F. Kottig, A hybrid framework combining data-driven and model-based methods for system remaining useful life prediction, Applied Soft Computing 44 (2016) 191-199.

13. B. Saha, K. Goebel and J. Christophersen, Comparison of prognostic algorithms for estimating remaining useful life of batteries, Transactions of the Institute of Measurement and Control 31(3-4) (2009) 293-308.

14. Z. Zhao, Q. Quan and K.-Y. Cai, A profust reliability based approach to prognostics and health management, IEEE Transactions on Reliability 63(1) (2014) 26-41.

15. R. Razavi-Far, S. Chakrabarti and M. Saif, Multi-step parallel-strategy for estimating the remaining useful life of batteries, in 2017 IEEE 30th Canadian Conf. on Electrical and Computer Engineering (CCECE) (2017), pp. 1-4.

16. B. Saha, K. Goebel, S. Poll and J. Christophersen, Prognostics methods for battery health monitoring using a bayesian framework, IEEE Trans. on Instrumentation and Measurement 58 (2009) 291-296.

17. R. Razavi-Far, S. Chakrabarti, M. Saif and E. Zio, An integrated imputationprediction scheme for prognostics of battery data with missing observations, Expert Systems with Applications 115 (2019) 709-723.

18. B. Saha and K. Goebel, Modeling li-ion battery capacity depletion in a particle filtering framework, in the Annual Conf. of the PHM Society (San Diego, CA, 2009).

19. D. Liu, Y. Luo, J. Liu, Y. Peng, L. Guo and M. Pecht, Lithium-ion battery remaining useful life estimation based on fusion nonlinear degradation AR model and RPF algorithm, Neural Computing and Applications 25(3-4) (2013) 557-572.

20. J. Liu, W. Wang and F. Golnaraghi, A multi-step predictor with a variable input pattern for system state forecasting, Mech. Syst. Signal Process 23 (2009) 1586-1599.

21. A. Saxena, J. Celaya, I. Roychoudhury, S. Saha, B. Saha and K. Goebel, Designing data-driven battery prognostic approaches for variable loading profiles: Some lessons learned, in European Conf. of Prognostics 85 Health Management (Dresden, Germany, 2012), pp. 1-11.

22. X. S. Si, An adaptive prognostic approach via nonlinear degradation modeling: Application to battery data, IEEE Transactions on Industrial Electronics 62(8) (2015) 5082-5096.

23. X. Xu, Z. Li and N. Chen, A hierarchical model for lithium-ion battery degradation prediction, IEEE Transactions on Reliability 65(1) (2016) 310-325.

24. X. Hu, J. Jiang, D. Cao and B. Egardt, Battery health prognosis for electric vehicles using sample entropy and sparse Bayesian predictive modeling, IEEE Transactions on Industrial Electronics 63(4) (2016) 2645-2656.

25. W. Wang, D. Wang, X. Wang, T. Li, R. Ahmed, S. Habibi and A. Emadi, Comparison of kalman filter-based state of charge estimation strategies for li-ion batteries, in 2016 IEEE Transportation Electrification Conf. and Expo (ITEC) (Dearborn, MI, 2016), pp. $1-6$.

26. M. Jouin, R. Gouriveau, D. Hissel, M. Péra and N. Zerhouni, Particle filter-based prognostics: Review, discussion and perspectives, Mechanical Systems and Signal Processing 72-73 (2016) 2-31,

27. C. S. K. Dash, P. Sahoo, S. Dehuri and S.-B. Cho, An empirical analysis of evolved radial basis function networks and support vector machines with mixture of kernels, International Journal on Artificial Intelligence Tools 24(4) (2015) 1550013. 
28. T. Böhm, Remaining useful life prediction for railway switch engines using classification techniques, International Journal of Prognostics and Health Management 8 (Special Issue 7) (2017).

29. R. G. Gore, J. Li, M. T. Manry, L. M. Liu, C. Yu and J. Wei, Iterative design of neural network classifiers through regression, International Journal on Artificial Intelligence Tools 14(1\&2) (2005) 281-301.

30. M. Ismail, R. Dlyma, A. Elrakaybi, R. Ahmed and S. Habibi, Battery state of charge estimation using an artificial neural network, in 2017 IEEE Transportation Electrification Conf. and Expo (ITEC) (Chicago, IL, 2017), pp. 342-349.

31. R. Razavi-Far, M. Farajzadeh-Zanjani, S. Chakrabarti and M. Saif, Data-driven prognostic techniques for estimation of the remaining useful life of lithium-ion batteries, in 2016 IEEE Int. Conf. on Prognostics and Health Management (ICPHM) (Ottawa, ON, 2016), pp. 1-8.

32. R. Razavi-Far, S. Chakrabarti and M. Saif, Multi-step-ahead prediction techniques for lithium-ion batteries condition prognosis, in 2016 IEEE Int. Conf. on Systems, Man, and Cybernetics (SMC) (2016), pp. 4675-4680.

33. Y. Liu, G. Zhao and X. Peng, A fusion prognostic approach based on multi-kernel relevance vector machine and Bayesian model averaging, in Proc. of 2016 Prognostics and System Health Management Conf. (PHM-Chengdu 2016) (Chengdu, 2016), pp. 1-6.

34. J. Yang, Z. Peng, H. Wang, H. Yuan and L. Wu, The remaining useful life estimation of lithium-ion battery based on improved extreme learning machine algorithm, International Journal of Electrochemical Science 13(5) (2018) 4991-5004.

35. G. B. Huang, H. Zhou, X. Ding and R. Zhang, Extreme learning machine for regression and multiclass classification, IEEE Transactions on Systems, Man, and Cybernetics Part B: Cybernetics 45(2) (2012) 513-529.

36. J. J. Carrasco, M. Millán-Giraldo, J. Caravaca, P. Escandell-Montero, J. M. MartínezMartínez and E. Soria-Olivas, ELM regularized method for classification problems, International Journal on Artificial Intelligence Tools 25(1) (2016) 1550026.

37. R. Razavi-Far, E. Hallaji, M. Saif and L. Rueda, A hybrid scheme for fault diagnosis with partially labeled sets of observations, in 2017 16th IEEE Int. Conf. on Machine Learning and Applications (ICMLA) (2017), pp. 61-67.

38. H. Takagi and I. Hayashi, Artificial neural network driven fuzzy reasoning, Int. J. Approx. Reason. 5(3) (1991) 191-212.

39. A. Hasiloglu, M. Yilmaz, O. Comakli and İsmail Ekmekci, Adaptive neuro-fuzzy modeling of transient heat transfer in circular duct air flow, Int. J. of Thermal Sciences 43(11) (2004) 1075-1090.

40. J.-S. Jang, ANFIS: Adaptive-network-based fuzzy inference system, IEEE Transactions on Systems, Man, and Cybernetics 23(3) (1993) 665-685.

41. A. Ivakhnenko, The group method of data handling in long-range forecasting, Technol. Forecast. Soc. Change 12(2-3) (1978) 213-217.

42. L. Breiman, Random forests, Machine Learning 45(1) (2001) 5-32.

43. H. Jaeger, The echo state approach to analyzing and training recurrent neural networks, Technical Report GMD Report 148, German National Research Center for Information Technology 148 (2001).

44. B. Saha and K. Goebel, Battery data set: Nasa ames prognostics data repository, in http://ti.arc.nasa.gov/project/prognostic-data-repository (NASA Ames, Moffett Field, CA, 2007).

45. S. Lee, H. Cui, M. Rezvanizaniani and J. Ni, Battery prognostics: SOC and SOH prediction, in Proc. of the ASME Int. Manufacturing Science and Engineering Conf. (Notre Dame, Indiana, USA, 2012), pp. 1-7. 
46. T. Chow and C. Leung, Nonlinear autoregressive integrated neural network model for short-term load forecasting, in IEE Procs. - Generation, Transmission and Distribution 143(5) (1996) 500-506.

47. S. Taieb, A. Sorjamaa and G. Bontempi, Multiple-output modeling for multi-stepahead time series forecasting, Neurocomputing 73(10-12) (2010) 1950-1957.

48. V. T. Tran, B.-S. Yang and A. C. C. Tan, Multi-step ahead direct prediction for the machine condition prognosis using regression trees and neuro-fuzzy systems, Expert Systems with Applications 36(5) (2009) 9378-9387.

49. R. Gouriveau and N. Zerhouni, Connexionist-systems-based long term prediction approaches for prognostics, IEEE Transactions on Reliability 61(4) (2012) 909-920.

50. Y. L. He, Z. Q. Geng and Q. X. Zhu, A data-attribute-space-oriented double parallel (DASODP) structure for enhancing extreme learning machine: Applications to regression datasets, Engineering Applications of Artificial Intelligence 41 (2015) 65-74.

51. A. Saxena, J. Celaya, B. Saha, S. Saha and K. Goebel, On applying the prognostic performance metrics, in Int. Conf. on Prognostics and Health Management (PHM) Annual Conf. of the Prognostics and Health Management Society (San Diego, USA, 2009). 